УДК 536.242

\title{
МОДЕЛИРОВАНИЕ ТЕЧЕНИЯ И ТЕПЛООБМЕНА В ГЛАДКИХ ТРУБАХ ПРИ СВЕРХКРИТИЧЕСКИХ ДАВЛЕНИЯХ
}

\author{
Фиалко Н.М. ${ }^{1}$, член-корреспондент НАН Украины, Пиоро И.Л. ${ }^{2}$, докт. техн. наук, Майсон Н.В. ${ }^{1}$, \\ Меранова Н.О. ${ }^{1}$, канд. техн. наук \\ ${ }^{1}$ Институт технической теплофизики НАН Украины, ул. Желябова, 2а, Киев, 03680, Украина \\ ${ }^{2}$ Faculty of Energy Systems and Nuclear Science University of Ontario Institute of Technology 2000 \\ Simcoe Str. N., Oshawa ON L1K 7 K4 Canada
}

Наводяться результати CFDмоделювання течії та теплообміну висхідних потоків води в гладких трубах при надкритичних тисках. Представлено дані щодо двовимірної картини локальних теплофізичних характеристик досліджуваних процесів. Розглядаються особливості руху фронту псевдокритичного переходу. Наводяться результати методичних досліджень 3 верифікації моделей турбулентності.

Библ. 18. рис. 7.
Приводятся результаты CFDмоделирования течения и теплообмена восходящих потоков воды в гладких трубах при сверхкритических давлениях. Представлены данные, касающиеся двумерной картины локальных теплофизических характеристик исследуемых процессов. Рассматриваются особенности движения фронта псевдокритического перехода. Приводятся результаты методических исследований по верификации моделей турбулентности.
CFD-simulation results of flow and heat transfer for upward water flow in bare tubes at supercritical pressure are given. The data relating to the two-dimensional picture of the local thermophysical characteristics of the investigated processes are presented. The features of the pseudocritical transition front movement are considered. The results of methodological studies on verification of turbulence models are discussed.

Ключевые слова: вычислительная гидродинамика, вода сверхкритического давления, псевдокритический фазовый переход, верификация моделей турбулентности.

$D$ - внутренний диаметр трубы, м;

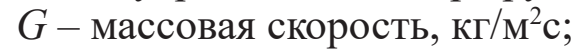

$L$ - обогреваемая длина трубы, м;

$P$ - давление, Па;

$q$ - плотность теплового потока, Вт/м²;

$r$ - радиальная координата, м;

$T$ - температура, ${ }^{\circ} \mathrm{C}$;

$T u$ - интенсивность турбулентности, \%;

$U_{z}$ - осевая компонента скорости, м/с;

$z$ - осевая координата, м;

$\alpha$ - коэффициент теплоотдачи, Вт/м² K.

\section{Нижние индексы:}

$\mathrm{dht}$ - ухудшенный теплообмен (deteriorated heat

\section{Введение}

Актуальные вопросы развития атомной энергетики непосредственно связаны с повышением тепловой эффективности АЭС. Одним из важных направлений такого повышения является приме- transfer);

in - входное сечение трубы (inlet);

pc - псевдокритический (pseudocritical);

\section{Сокращения:}

AKN - Abe-Kondoh-Nagano $k$ - $\varepsilon$ turbulent model;

BSL - Baseline $k-\omega$ turbulent model;

CFD - Computational Fluid Dynamics;

LB - Lam-Bremhorst $k$ - $\varepsilon$ turbulent model;

NIST - National Institute of Standards and Technology;

REFPROP - REFerence PROPerties;

SST - Shear Stress Transport turbulent model;

АЭС - атомная электростанция.

нение в качестве теплоносителей ядерных реакторов жидкостей при сверхкритических давленияX.

В комплексе научно-технических проблем, касающихся реакторов на сверхкритических 
давлениях, большое значение имеют задачи реакторной теплофизики. Анализ состояния исследований в области реакторной теплофизики при сверхкритических давлениях представлен в ряде работ [1-5]. Одна из тенденций данных исследований состоит в повышении роли CFDмоделирования для предсказания закономерностей течения и теплообмена сверхкритических жидкостей (например, [6-11]). При этом такие предсказания становятся все более информативными и достоверными. К тому же они позволяют получать многомерные картины локальных теплофизических характеристик процесса.

Цель данной работы состоит в исследовании на основе CFD-моделирования двумерной картины течения и теплообмена сверхкритической воды при подъемном течении в гладких трубах.

\section{Постановка задачи и методика исследований}

Как объект теплофизических исследований рассматриваемая ситуация отвечает задаче смешанной теплоотдачи (при вынужденном и свободном движении) в условиях существенной зависимости свойств жидкости от температуры. В работе данная задача решалась в двумерной осесимметричной постановке. С целью стабилизации течения перед входом в трубу расчетная область увеличивалась вверх по потоку за счет начального необогреваемого участка длиной 1,2 м. Во входном сечении трубы массовая скорость $G$ и температура $T_{\text {in }}$ принимались постоянными, а величина интенсивности турбулентности $T u$ - равной 3 \%. В выходном сечении трубы ставились «мягкие» граничные условия. На обтекаемых водой поверхностях трубы задавались условия прилипания. На необогреваемом участке трубы, в качестве тепловых, принимались условия адиабатичности, на обогреваемом участке - условия постоянного по длине трубы теплоподвода к ее стенке. Расчетная область покрывалась неравномерной сеткой с существенным сгущением к стенкам трубы и содержала $120 \times 520$ ячеек. Пристеночный шаг задавался равным $1,5 \cdot 10^{-6} \mathrm{M}$, что обеспечивало значение $\mathrm{y}^{+}<0,7\left(\mathrm{y}^{+}=u_{\tau} y_{1} / v\right.$, где $u_{\tau}-$ динамическая скорость, $y_{1}$ - радиальное расстояние от стенки до первого пристеночного узла сетки, $v$ - локальная кинематическая вязкость). Для определения физических свойств сверхкритической воды использовалась программа NIST REFPROP, интегрированная в FLUENT код. Решение поставленной задачи осуществлялось с double precision.

В работе выполнена верификация моделей турбулентности путем сопоставления известных экспериментальных данных и численных решений, полученных с использованием различных моделей турбулентности, которые фигурируют в современном каталоге замыкающих моделей. Рассматривались модели, относящиеся к трем следующим группам: высокорейнольдсовые $k$ - $\varepsilon$ модели с пристеночными функциями, низкорейнольдсовые $k$ - $\varepsilon$ модели и $k-\omega$ модели. Из моделей первой группы тестировались Realizable [12] и Standard $k-\varepsilon$ модели [13], из моделей второй группы $k-\varepsilon$ AKN [14] и $k$ - $\varepsilon$ LB [15], из моделей третьей группы - $k$ - $\omega$ SST и $k$ - $\omega$ BSL [16]. На рис. 1 в качестве примера представлены результаты соответствующих сопоставлений с экспериментальными данными, отвечающими следующим параметрам: массовая скорость $G=1002$ кг/ $\mathbf{M}^{2} \mathrm{c}$, плотность подводимого к стенке теплового потока $q=681$ кВт/м², давление на входе в трубу $P_{\text {in }}=23,9$ МПа, температура во входном сечении трубы $T_{\text {in }}=350{ }^{\circ} \mathrm{C}$ [5].

Из анализа данных, приведенных на рис. 1, следует, что рассмотренные модели турбулентности по убыванию адекватности описания ими экспериментальных данных ранжируются следующим образом: $k-\omega \mathrm{SST}, k-\varepsilon$ Standard, $k-\omega$ BSL, $k-\varepsilon$ Realizable, $k-\varepsilon$ AKN и $k-\varepsilon$ LB. При этом для трех последних моделей турбулентности из этого списка расхождения экспериментальных и расчетных данных являются недопустимо большими. То есть рассмотренные низкорейнольдсовые модели и высокорейнольдсовая модель $k$ - $\varepsilon$ Realizable не в полной мере отвечают анализируемой физической ситуации. Что же касается первых трех моделей, то здесь отклонения экспериментальных и расчетных данных в целом сравнительно невелики. Эти отклонения являются наибольшими на начальном обогреваемом участке трубы и наименьшими - вблизи выхода из нее. Первые две модели из приведенного списка соотносятся между собой следующим образом. Модель $k$ - $\varepsilon$ Standard существенно уступает по адекватности модели $k-\omega$ на значительном по протяженности центральном участке трубы. 
Данные, полученные на основе этих моделей, практически совпадают вблизи выхода из трубы. Модель $k$ - $\varepsilon$ имеет некоторые преимущества лишь на начальном обогреваемом участке. Таким образом, рассматриваемой физической ситуации в наибольшей мере отвечает $k-\omega$ SST модель турбулентности.

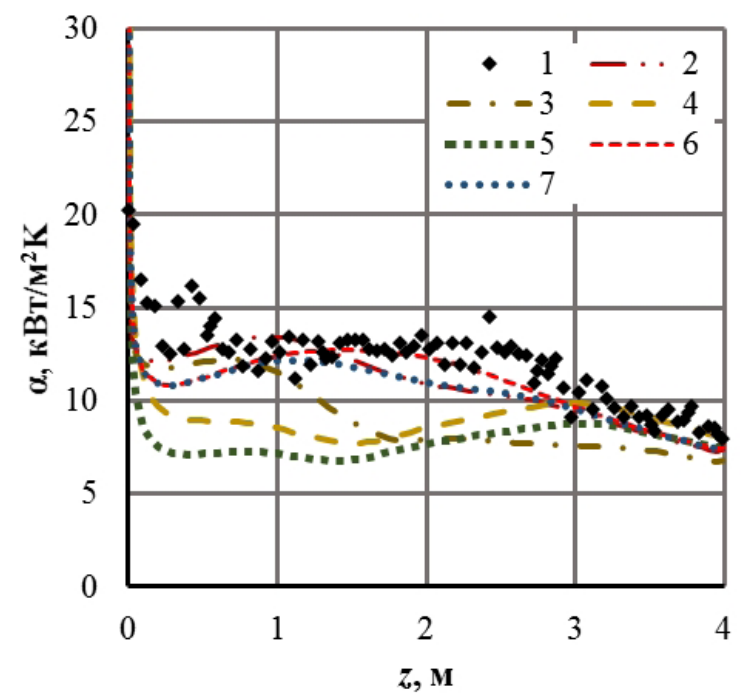

a)
Характеризуя в целом полученные данные по тестированию моделей турбулентности, следует отметить, что для всех исследуемых моделей имеет место тенденция к определенному завышению данных по температуре стенки и соответственно к занижению значений коэффициентов теплоотдачи.

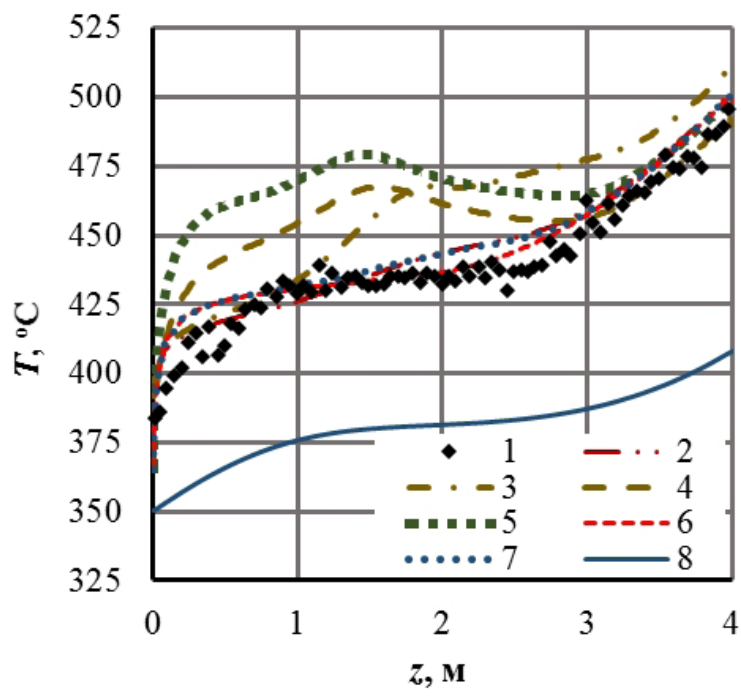

б)

Рис. 1. Сопоставление экспериментальных (1) и расчетных (2-7) данных для распределения коэффициента теплоотдачи (а) и температуры внутренней поверхности стенки и среднемассовой температуры (8) воды (б) вдоль обогреваемого участка трубы при использовании различных моделей турбулентности:

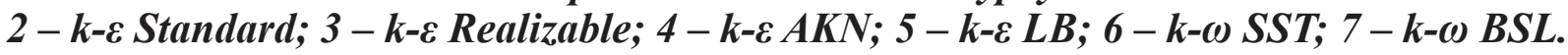

\section{Результаты исследований и их анализ}

Ниже приводятся результаты CFDмоделирования, полученные с использованием $k-\omega \mathrm{SST}$ модели турбулентности. Основные исходные данные для анализируемой ситуации отвечают следующим значениям: $G=1002$ кг $/ \mathrm{M}^{2} \mathrm{c}$; $q=581 \kappa \mathrm{\kappa \textrm {T }} / \mathrm{M}^{2} ; P_{\text {in }}=23,9 \mathrm{M \Pi а} ; T_{\text {in }}=350^{\circ} \mathrm{C}$; $D=10 \mathrm{мm} ; L=4$ м. Рассчитанная по зависимости [17] величина минимального теплового потока $q_{d h t}$, при превышении которого возникает режим ухудшенного теплообмена, в рассматриваемых условиях составляет $687 \mathrm{KBT} / \mathrm{M}^{2}$, что больше заданного значения подводимого теплового потока. То есть в исследуемой ситуации режим ухудшенного теплообмена отсутствует.

Рассмотрим основные закономерности протекания исследуемого процесса. Эти закономерности связаны со следующими известны- ми особенностями течения и теплообмена при сверхкритических давлениях [18]. Во-первых, в данных условиях имеет место резкое изменение теплофизических свойств теплоносителя с температурой. Во-вторых, наблюдается ускорение потока в результате уменьшения плотности жидкости по длине обогреваемого канала. И наконец, в ряде ситуаций развивается естественная конвекция за счет архимедовых сил, обусловленных разницей плотностей жидкости в различных точках сечения потока.

Ниже полученные данные анализируются в аспекте указанных основных особенностей течения и теплообмена при сверхкритических давлениях.

Остановимся вначале на рассмотрении особенностей течения сверхкритической воды. На рис. 2 представлены профили скорости в ради- 
альном направлении на различном расстоянии от входа. Как видно, имеет место существенное ускорение потока по длине трубы. Что же касается формы профилей скорости, то они представляют собой усеченные параболы и не содержат участков с повышенными значениями скорости вблизи стенки трубы. Это свидетельствует о том, что в рассматриваемых условиях влияние подъемных сил пренебрежимо мало.

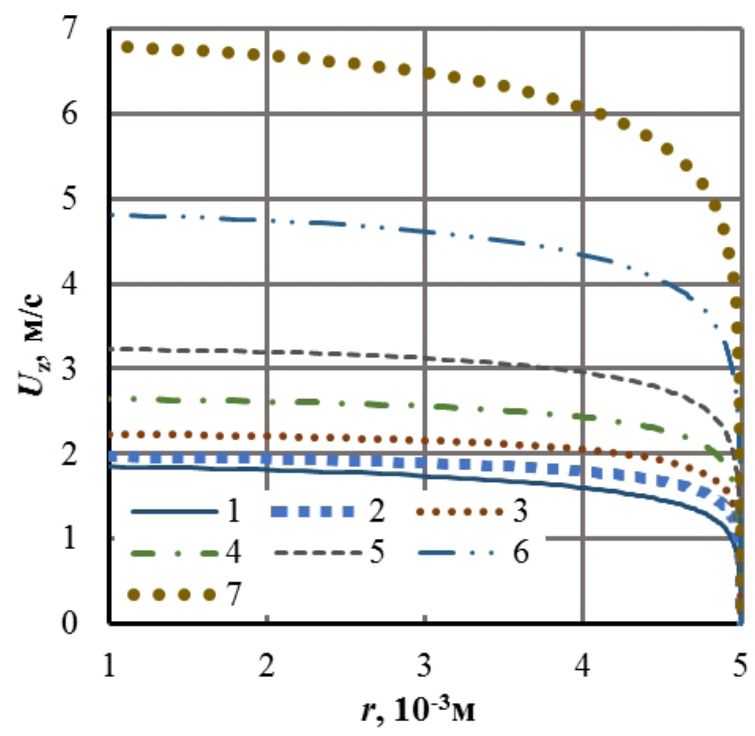

Рис. 2. Профили осевой компоненты скорости в поперечном сечении трубы на различном удалении zот входа в ее обогреваемый участок:

$1-z=0,0 \mathrm{~m} ; 2-z=0,5 \mathrm{~m} ; 3-z=1,0 \mathrm{~m} ; 4-z=1,5 \mathrm{~m} ; 5-z=2,0 \mathrm{~m} ; 6-z=3,0 \mathrm{~m} ; 7-z=4,0 \mathrm{~m}$.

Согласно полученным данным форма профилей скорости несколько трансформируется по длине трубы. А именно, меняется степень заполненности данных профилей. По мере удаления от входа в обогреваемый участок трубы указанная степень заполненности возрастает. И далее, начиная с расстояния от входа равного примерно 2 метрам, она заметно снижается.

Рисунок 3 иллюстрирует изменение по длине трубы осевой компоненты скорости $U_{z}$ при различных значениях $r$. Как следует из приведенных данных, наибольшее повышение скорости имеет место на оси трубы $(r=0)$ и уменьшается по мере приближения к ее стенке. Так, скорость $U_{z}$ на оси увеличивается от входа к выходу трубы примерно на $5 \mathrm{~m} / \mathrm{c}$, а на расстоянии одной сотой миллиметра от стенки трубы - лишь на 2 м/с. Важно также отметить, что поток ускоряется по длине трубы весьма неравномерно. Данное ускорение заметно повышается вниз по потоку, начиная примерно с середины длины трубы. При этом указанная тенденция проявляется более существенно при при- ближении к оси трубы.

Рассмотрим далее закономерности теплообмена. На рис. 4 представлено сопоставление экспериментальных и расчетных данных об изменении по длине обогреваемого участка трубы коэффициента теплоотдачи и температуры стенки трубы. Приведенные данные иллюстрируют вполне удовлетворительное согласование результатов экспериментов и расчетов.

Как видно из рис. 4, поведение коэффициента теплоотдачи характеризуется тем, что его изменение в центральной по длине части трубы оказывается в целом относительно незначительным. То есть наблюдается картина, подобная стабилизации теплообмена в трубах при докритических параметрах жидкости. На удалении примерно 2,5 м от входа в обогреваемый участок трубы начинается падение коэффициента теплоотдачи, обусловленное в большой мере образованием вблизи стенки трубы слоя, препятствующего переносу тепла, в котором турбулентная теплопроводность весьма мала. 
Характер изменения температуры стенки коррелируется определенным образом с поведением коэффициента теплоотдачи. Здесь также на центральном по длине участке трубы температура $T_{\text {wall }}$ остается практически постоянной. При этом осевая координата, отвечающая началу заметного повышения температуры $T_{\text {wall }}$, совпадает с координатой, соответствующей началу снижения $\alpha$.
Что касается среднемассовой температуры жидкости $T_{b u l k}$, то характер ее изменения качественно аналогичен поведению температуры стенки. Однако для $T_{b u l k}$ осевая координата начала и конца участка, на котором она остается практически постоянной, несколько смещается вниз по потоку.

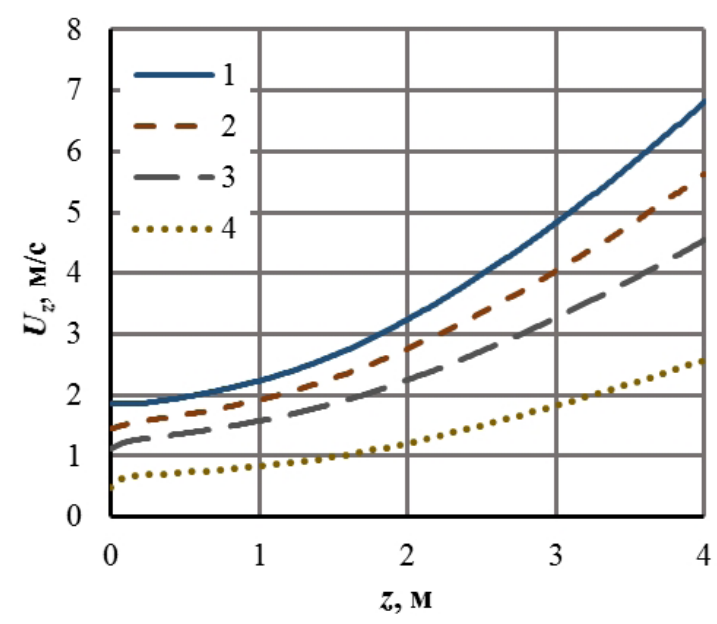

Рис. 3. Распределение осевой компоненты скорости в продольном направлении трубы на различном удалении г от ее оси:

$1-r=0,0 \mathrm{~m} ; 2-r=4,50 \cdot 10^{-3} \mathrm{M} ; 3-r=4,90 \cdot 10^{-3} \mathrm{M} ; 4-r=4,99 \cdot 10^{-3} \mathrm{M}$.

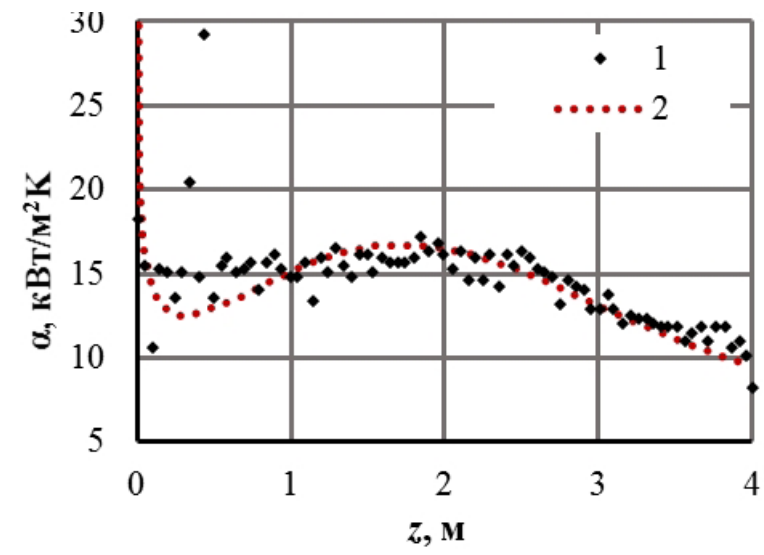

a)

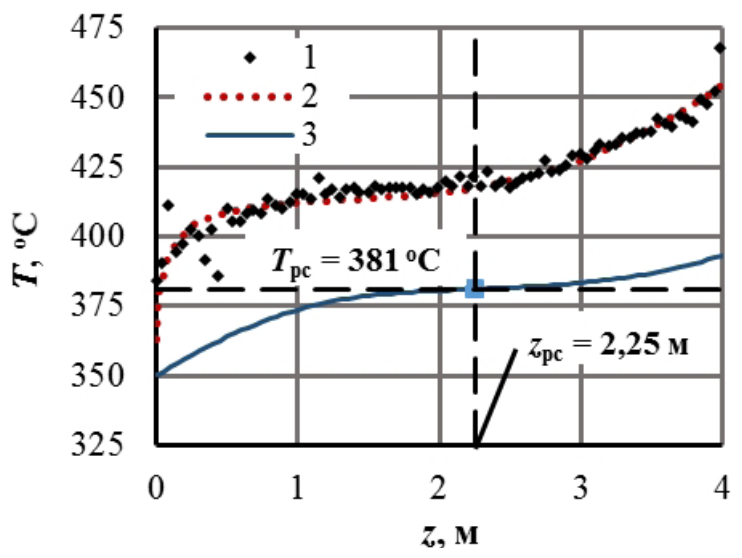

б)

Рис. 4. Сопоставление экспериментальных (1) и расчетных (2) данных для распределения коэффициента теплоотдачи (a) и температуры внутренней поверхности стенки и среднемассовой температуры (3) воды (б) вдоль обогреваемого участка трубы.

Рис. 5 иллюстрирует радиальные профили температуры на различном расстоянии от входа в обогреваемую часть трубы. Согласно приведенным данным конфигурация этих профилей изме- няется по длине трубы таким же образом, как и профилей скорости. То есть с удалением от входа в трубу заполненность профилей увеличивается и затем примерно от середины трубы уменьшается. 


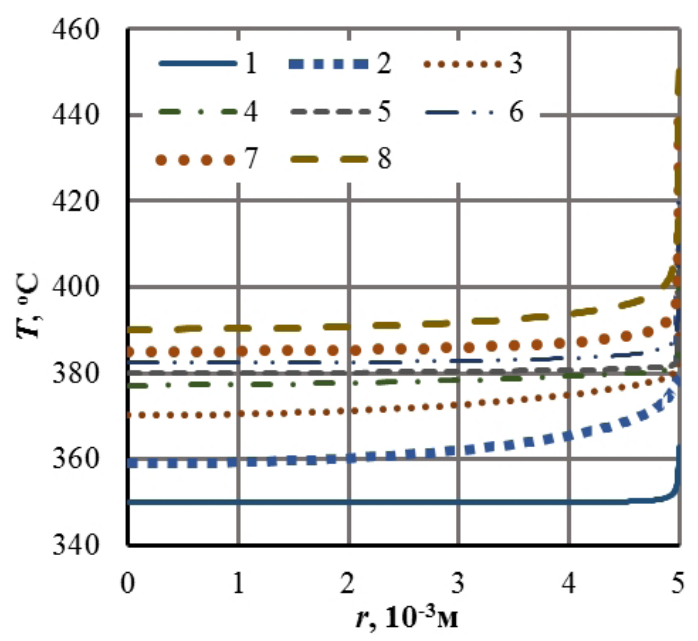

Рис. 5. Профили температуры в поперечном сечении трубы на различном удалении $z$ от входа в ее обогреваемый участок: $1-z=0,0 \mathrm{~m} ; 2-z=0,5 \mathrm{~m} ; 3-z=1,0 \mathrm{~m}$;

$$
4-z=1,5 \mathrm{~m} ; 5-z=2,0 \mathrm{~m} ; 6-z=3,0 \mathrm{~m} ; 7-z=3,5 \mathrm{M} ; 8-z=4,0 \mathrm{M} \text {. }
$$

Изменение температуры жидкости по длине трубы в фиксированных точках $r=$ const показано на рис. 6. Как видно из рис. 6 и рис. 4 , температура жидкости вблизи стенки $\left(r=4,95 \cdot 10^{-3} \mathrm{M}\right)$ следует за температурой стенки. Ее изменение характеризуется наличием достаточно про- тяженного по потоку участка, на котором она остается практически постоянной. В точках более удаленных от стенки трубы данный участок сокращается. Причем его протяженность тем меньше, чем ближе к оси трубы расположена рассматриваемая точка.

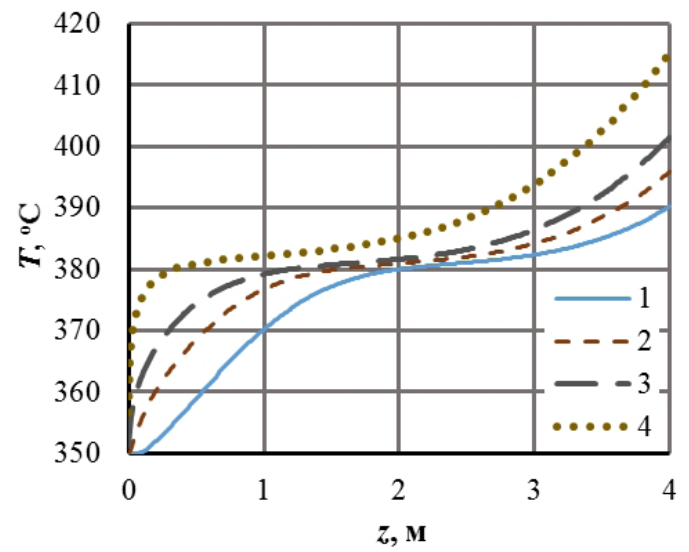

Рис. 6. Распределение температуры в продольном направлении трубы при различном удалении r от ее оси: $1-r=0,0 \mathrm{~m} ; 2-r=4,50 \cdot 10^{-3} \mathrm{M} ; 3-r=4,90 \cdot 10^{-3} \mathrm{M} ; 4-r=4,99 \cdot 10^{-3} \mathrm{M}$.

На рис. 7 приведены данные о положении фронта псевдокритического перехода. (Здесь положение фронта фиксируется по псевдокритической температуре $T_{p c}=381{ }^{\circ} \mathrm{C}$, которая определяется как температура, отвечающая максимуму теплоемкости). Как следует из приведенных данных, примерно до середины обогреваемого участка трубы фронт движется от ее стенки дос- таточно медленно. Далее вниз по потоку движение фронта ускоряется, так что на расстоянии 2,46 м от входа в обогреваемый участок трубы он достигает ее оси. Очевидно, среднемассовая температура жидкости $T_{b u l k}$ должна достигать значения, равного $T_{p c}$, несколько выше по потоку. Как видно из рис. 4 , температура $T_{b u l k}$ становится равной $T_{p c}$ на расстоянии 2,25 м от входа в трубу. 


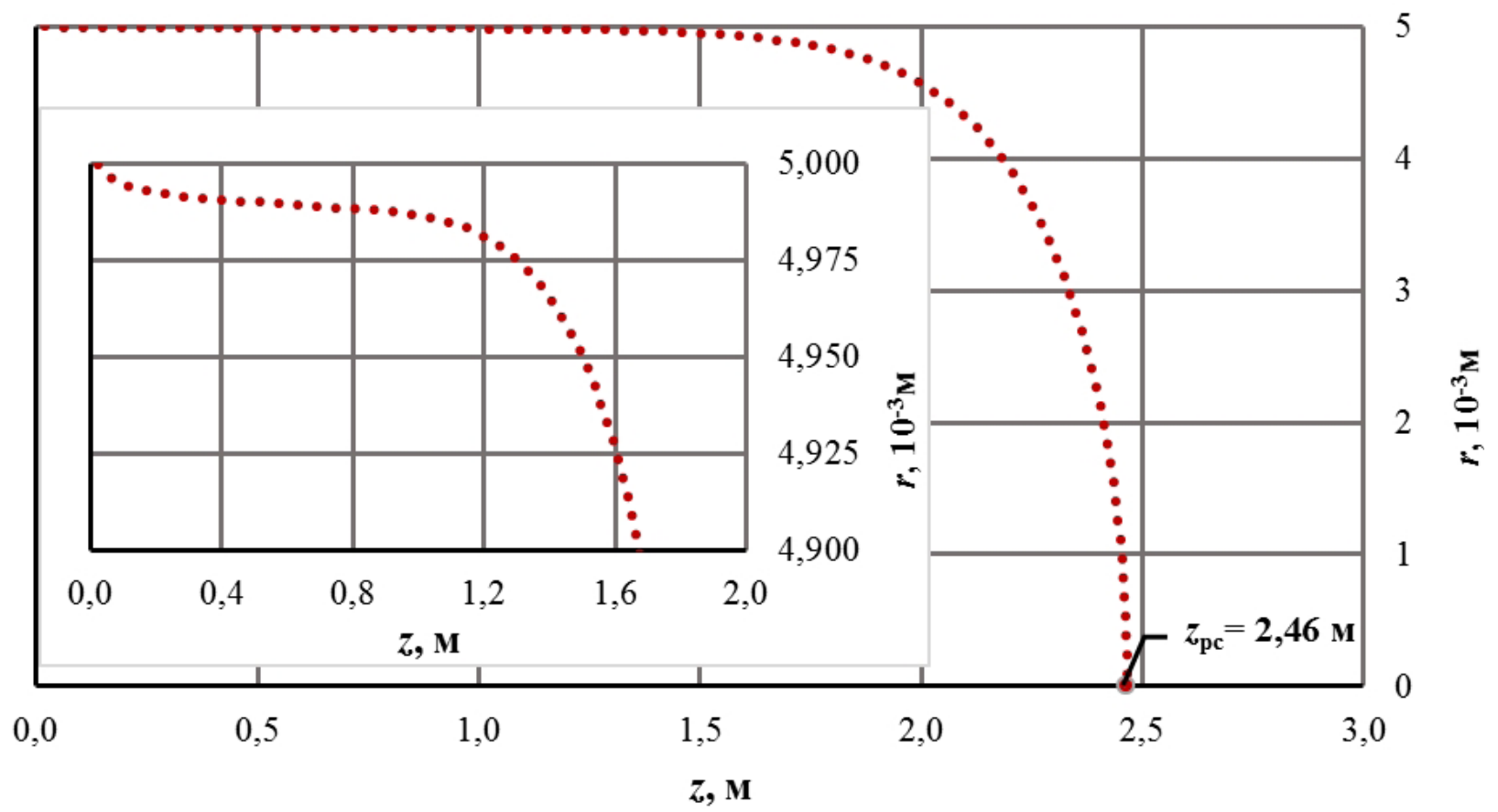

Рис. 7. Конфигурация фронта псевдокритического фазового перехода воды в трубе.

\section{Выводы}

1. Выполнены численные исследования характеристик течения и теплообмена сверхкритической воды в вертикальных гладких трубах с нагреваемой длиной 4 м и внутренним диаметром 10 мм при массовой скорости жидкости $G=1002$ кг/м²c. Проведено тестирование моделей турбулентности из трех следующих групп: высокорейнольдсовые $k$ - $\varepsilon$ модели с пристеночными функциями, $k$ - $\omega$ модели и низкорейнольдсовые $k$ - $\varepsilon$ модели. На основе сопоставления результатов экспериментальных и расчетных исследований показано, что наименьшие отклонения сравниваемых данных имеют место для $k$ - $\omega$ SST модели турбулентности.

2. Установлены основные особенности двумерной картины течения и теплообмена для исследуемых условий. Особое внимание уделено рассмотрению закономерностей изменения характеристик потока сверхкритической воды по радиусу трубы. Представлены данные о конфигурации фронта псевдокритического перехода.

\section{ЛИТЕРАТУРА}

1. Bazargan $M$. Forced convection heat transfer to turbulent flow of SCW in a round horizontal tube // PhD Thesis, University of British Columbia, Canada. - 2001. -340 p.

2. Pioro I.L., Khartabil H.F., Duffey, R.B. Literature survey devoted to the heat transfer and hydraulic resistance of fluids at supercritical pressures and near critical pressures // AECL Report. 2002. $-190 \mathrm{p}$.

3. Pioro I., Duffey $R$. Heat Transfer and Hydraulic Resistance at Supercritical Pressures in Power Engineering Applications // ASME Press, New York, NY, USA. - 2007. - 334 p.

4. Hussam A. M. Zahlan, Derivation of a lookup table for Transcritical heat transfer in water-cooled tubes // PhD Thesis, Ottawa-Carleton Institute for Mechanical and Aerospace Engineering Faculty of Engineering University of Ottawa, Canada. - 2015. $-252 \mathrm{p}$.

5. Kirillov P., Pometko R., Smirnov A., Grabezhnaia $V$. Experimental Study on Heat Transfer to Supercritical Water Flowing in 1- and 4-m-Long Vertical Tubes // Proc. GLOBAL'05, Tsukuba, Japan. - 2005.- p. 518.

6. Agranat V., Malin M., Pioro I., Abdullah R., Perminov V.A. CFD Modelling of Supercritical Water Heat Transfer in a Vertical Bare Tube Upward Flow // Proceedings of ICONE-23, May 17-21, 
Chiba, Japan. - 2015. - Paper 1163. - 11 p.

7. Cheng X., Kuang B., Yang Y.H. Numerical analysis of heat transfer in supercritical water cooled flow channels // Nuclear Engineering and Design. - 2007. - Vol. 237. - pp.240-252.

8. Farah A., Harvel G., Pioro I. Assessment of Fluent CFD Code as an Analysis Tool for Supercritical-Water Heat-Transfer Applications // Proceedings of the 15th International Topical Meeting on Nuclear Reactor Thermalhydraulics (NURETH-15), Pisa, Italy, May 12-15, 2013. Paper 118. - $13 \mathrm{p}$.

9. Koshizuka S., Takano N., Oka Y. Numerical Analysis of Deterioration Phenomena in Heat Transfer to Supercritical Water // Int. J. Heat Mass Transfer. - 1995. - Vol. 38. - pp.3077-3084.

10. Yang J., Oka Y., Ishiwatari Y, Liu J., Yoo J. Numerical investigation of heat transfer in upward flows of supercritical water in circular tubes and tight fuel rod bundles // Nuclear Engineering and Design. - 2007. - Vol. 237. - pp.420-430.

11. Shang Z. CFD investigations of vertical rod bundles of supercritical water-cooled nuclear reactor // Nuclear Engi-neering and Design. - 2009, Vol. 239. - pp. $2562-2572$.

12. Shih T.H., Liou W.W., Shabbir A., Yang $Z$., Zhu J. A new k-epsilon eddy viscosity model for high Reynolds number turbulent flows: Model development and validation // Computers and Fluids. - 1994. - Vol. 24(3). - pp. 227-238.

13. Launder B.E., Spalding, D.B. The numerical computation of turbulent Flows // Computer Methods in Applied Mechanics and Engineering. 1974. - Vol. 3(2). - pp.269-289.

14. Abe K., Kondoh T., Nagano Y. A new turbulence model for predicting fluid flow and heat transfer in separating and reattaching flows I. flow field calculations // International Journal of Heat and Mass Transfer. - 1994. - Vol. 37(1). pp.139-151.

15. Lam C.K.G., Bremhorst K. A modified form of the k-epsilon model for predicting wall turbulence // ASME Journal of Fluids Engineering. - 1981. Vol. 103. - pp.456-460

16. Menter F.R. Two-equation eddy-viscosity turbulence models for engineering applications // AIAA Journal. - 1994. - Vol. 32(8). - pp.1598-1605.

17. Mokry S., Pioro I.L., Farah A., King K., Gupta S., Peiman W., Kirillov P. Development of Supercritical Water Heat-Transfer Correlation for Vertical Bare Tubes // Nuclear Engineering and Design. - 2011. - Vol. 241. - pp. 1126-1136.

18. Грабежная В.А., Кириллов П.Л. О расчетах теплообмена в трубах и пучках стержней при течении воды сверхкритического давления // Обзор ФЭИ-0297. Цнииатоминформ, 2003. 


\section{SIMULATION OF FLOW AND HEAT TRANSFER IN BARE TUBES AT SUPERCRITICAL PRESSURE}

\section{Fialko N.M. ${ }^{1}$, Pioro I.L. ${ }^{2}$, Maison N.V. ${ }^{1}$, Meranova N.O. ${ }^{1}$}

${ }^{1}$ Institute of Engineering Thermophysics of the National Academy of Sciences of Ukraine, Zhelyabova 2a, Kyiv, 03057, Ukraine

${ }^{2}$ Faculty of Energy Systems and Nuclear Science University of Ontario Institute of Technology 2000 Simcoe Str. N., Oshawa ON L1K 7K4 Canada

The paper is devoted to the numerical simulation of water flow and heat transfer in bare tubes at supercritical pressures. The studies were carried out using Fluent software for upward flow in a circular tube with a heated length of $4 \mathrm{~m}$ and an inner diameter of $10 \mathrm{~mm}$ at water mass flux $G=1002 \mathrm{~kg} / \mathrm{m}^{2} \mathrm{~s}$. The data are presented with respect to verification turbulence models for the considered physical situation. This testing includes different turbulence models appearing in modern catalog of these models. Namely, the models related to the following three groups were considered: high-Re $k-\varepsilon$ model with wall functions, low-Re $k-\omega$ models and $k-\varepsilon$ models. It is shown that the best agreement for known experimental data with the results of computer simulation answers $k-\omega \mathrm{SST}$ turbulence model. The analysis of the main features of the two-dimensional flow patterns and heat transfer for the considered conditions is performed. Particular attention is paid to the discussion of the research results concerning the features of the thermal-hydraulic parameters in the cross sections of the tube. The data on the nature of the pseudocritical transition front motion in the tube are given. References 18, figures 7.

1. Bazargan $M$. Forced convection heat transfer to turbulent flow of SCW in a round horizontal tube // PhD Thesis, University of British Columbia, Canada. - 2001. - 340 p. (Eng.)

2. Pioro I.L., Khartabil H.F., Duffey, R.B. Literature survey devoted to the heat transfer and hydraulic resistance of fluids at supercritical pressures and near critical pressures // AECL Report.
- 2002. - 190 p. (Eng.)

3. Pioro I., Duffey R. Heat Transfer and Hydraulic Resistance at Supercritical Pressures in Power Engineering Applications // ASME Press, New York, NY, USA. - 2007. - 334 p. (Eng.)

4. Hussam A. M. Zahlan. Derivation of a look-up table for Trans-critical heat transfer in water-cooled tubes // PhD Thesis, Ottawa-Carleton Institute for Mechanical and Aerospace Engineering Faculty of Engineering University of Ottawa, Canada. - 2015. -252 p. (Eng.)

5. Kirillov P., Pometko R., Smirnov A., Grabezhnaia $V$. Experimental Study on Heat Transfer to Supercritical Water Flowing in 1- and 4-m-Long Vertical Tubes // Proc. GLOBAL'05, Tsukuba, Japan. - 2005.- p. 518. (Eng.)

6. Agranat V., Malin M., Pioro I., Abdullah R., Perminov V.A. CFD Modelling of Supercritical Water Heat Transfer in a Vertical Bare Tube Upward Flow // Proceedings of ICONE-23, May 17-21, Chiba, Japan. - 2015. - Paper 1163. - 11 p. (Eng.)

7. Cheng X., Kuang B., Yang Y.H. Numerical analysis of heat transfer in supercritical water cooled flow channels // Nuclear Engineering and Design. 2007. - Vol. 237. - pp.240-252. (Eng.)

8. Farah A., Harvel G., Pioro I. Assessment of Fluent CFD Code as an Analysis Tool for Supercritical-Water Heat-Transfer Applications // Proceedings of the 15th International Topical Meeting on Nuclear Reactor Thermalhydraulics (NURETH-15), Pisa, Italy, May 12-15, 2013. Paper 118. - 13 p. (Eng.)

9. Koshizuka S., Takano N., Oka Y. Numerical Analysis of Deterioration Phenomena in Heat Transfer to Supercritical Water // Int. J. Heat Mass Transfer. - 1995. - Vol. 38. - pp.3077-3084. (Eng.)

10.Yang J., Oka Y., Ishiwatari Y, Liu J., Yoo J. Numerical investigation of heat transfer in upward flows of supercritical water in circular tubes and tight fuel rod bundles // Nuclear Engineering and Design. - 2007. - Vol. 237. - pp.420-430. (Eng.)

11. Shang Z. CFD investigations of vertical rod bundles of supercritical water-cooled nuclear reactor // Nuclear Engi-neering and Design. - 2009, Vol. 239. - pp. 2562 - 2572. (Eng.)

12.Shih T.H., Liou W.W., Shabbir A., Yang Z., Zhu J. A new k-epsilon eddy viscosity model for high Reynolds number turbulent flows: Model 
development and validation // Computers and Fluids. - 1994. - Vol. 24(3). - pp. 227-238. (Eng.)

13. Launder B.E., Spalding, D.B. The numerical computation of turbulent Flows // Computer Methods in Applied Mechanics and Engineering. - 1974. Vol. 3(2). - pp.269-289. (Eng.)

14. Abe K., Kondoh T., Nagano Y. A new turbulence model for predicting fluid flow and heat transfer in separating and reattaching flows - I. flow field calculations // International Journal of Heat and Mass Transfer. - 1994. - Vol. 37(1). - pp.139-151. (Eng.)

15. Lam C.K.G., Bremhorst K. A modified form of the k-epsilon model for predicting wall turbulence // ASME Journal of Fluids Engineering. -
1981. - Vol. 103. - pp.456-460. (Eng.)

16. Menter F.R. Two-equation eddy-viscosity turbulence models for engineering applications // AIAA Journal. - 1994. - Vol. 32(8). - pp.1598-1605. (Eng.)

17. Mokry S., Pioro I.L., Farah A., King K., Gupta S., Peiman W., Kirillov P. Development of Supercritical Water Heat-Transfer Correlation for Vertical Bare Tubes // Nuclear Engineering and Design. - 2011. - Vol. 241. - pp. 1126-1136. (Eng.) 18. Grabehznaia, V.A., Kirillov, P.L About Calculation of Heat Transfer in the Tubes and Bundles of Rods at Flow of Supercritical Pressure Water // Obzor FEI-0297. Tsniiatominform. 2003. (Rus.) 